\title{
ORIGINAL ARTICLE \\ Does consumption of polyunsaturated fatty acids influence on neurorehabilitation in traumatic spinal cord-injured individuals? a double-blinded clinical trial
}

\author{
A Norouzi Javidan ${ }^{1}$, H Sabour ${ }^{1}$, S Latifi ${ }^{1}$, M Abrishamkar ${ }^{1}$, Z Soltani ${ }^{1}$, F Shidfar ${ }^{2}$ and H Emami Razavi ${ }^{1}$
}

Study design: A double-blinded randomized clinical trial.

Objectives: The anti-inflammatory and neuroprotective effect of omega- 3 fatty acids have been shown so far, but still its influence on clinical measures in spinal cord-injured human models were not known. We tried to investigate changes in disability and dependency scores in chronic traumatic spinal cord-injured patients after 14 months of $\omega-3$ fatty-acid consumption.

Methods: Main inclusion criteria were: traumatic spinal cord injury (SCI) and post injury duration longer than 1 year. Disability and dependency was assessed using UK Functional Independence Measure and Functional Assessment Measure (FIM + FAM) scale. MorDHA capsules ( $435 \mathrm{mg}$ of docosahexaenoic acid and $65 \mathrm{mg}$ of eicosapentaenoic acid) were administered in treatment group, whereas control group received placebo capsules for 14 months. UK FIM + FAM scale were estimated before intervention and at the end of the trial.

Results: Fifty-four patients in treatment group and 50 patients in placebo group completed the trial. Highest scores were detected in cognitive domain in both groups before and after intervention. Most dependency was observed in locomotion subscale and secondly in sphincter control. Scores of none of these components were changed by $\omega$ - 3 fatty-acid consumption.

Conclusion: Although omega-3 fatty acids have been shown to have neuroprotective effect in acute phase of $\mathrm{SCl}$, it seems that they have no significant influence in chronic inflammatory state of $\mathrm{SCl}$. The positive effect of $\omega-3$ fatty acid in chronic neurorecovery process, if exists, is weaker to exert any significant improvement in UK FIM + FAM scores in spinal cord-injured individuals. Spinal Cord (2014) 52, 378-382; doi:10.1038/sc.2014.30; published online 18 March 2014

Keywords: fatty acids; omega-3; rehabilitation; spinal cord injury

\section{INTRODUCTION}

Spinal cord injury (SCI) imposes a high burden of immobility and decreased life quality on involved individuals ${ }^{1}$ and induction of neuroprotection and neurorecovery has been always challenging in this field. The annual incidence of SCI is about 40 cases per million in United States ${ }^{2}$ and is estimated to be about 10-15 cases per million in United Kingdom. ${ }^{2}$ It seems that incidence of SCI is higher in developing countries. Although no specific report of precise incidence of SCI could be found in Iran, a recent study revealed the prevalence of SCI in Tehran, which currently stands at 1.2-11.4 per 10000 people. $^{3}$ Recently, some reports have supported the positive effect of omega- 3 polyunsaturated fatty acids ( $\omega-3$ PUFAs) in induction of neurorecovery, ${ }^{4,5}$ which is mediated through its antioxidant and anti-inflammatory effects. ${ }^{6}$ PUFAs are important components of phospholipids which contribute in constitution of cell membrane. Omega 3 PUFAs consist of $\alpha$-linolenic acid, eicosapentaenoic acid and docosahexaenoic acid. Although $\alpha$-Linolenic acid represents biosynthesis, the exogenous forms of the other two components are available. Various investigations have evaluated neuroprotective effects of $\omega-3$ PUFAs in animal models, while studies on human are limited. In this study, we tried to investigate the influence of $\omega-3$ PUFAs consumption on neurorehabilitation in patients with chronic SCI.

The purpose of this study was to investigate whether consumption of $\omega$-3 PUFAs has any effects on outcomes of disability measures in patients with SCI. We tried to understand whether administration of (-3 PUFAs may reveal any positive effect on Functional Independence Measure (FIM) and Functional Assessment Measure (FAM; FIM + FAM) scores in these patients.

\footnotetext{
MATERIALS AND METHODS

Participants

Participants in this clinical trial, which was designed as a placebo-controlled investigation, (registration number: NCT01311375: 6 March 2011) were chronic spinal cord-injured patients who were referred to Brain and Spinal Injury Research Center. An adequate explanation about trial was given to each individual before enrolling and written consent was obtained from each patient. At the end of the study, 104 patients completed the 14 months followup: fifty-four patients in treatment group and 50 patients in placebo group (six patients did not complete the study protocol) (Figure 1). The two most common reasons for drop out were experiencing gastrointestinal side effects or difficulty to maintain scheduled clinical visits.
}

${ }^{1}$ Brain and Spinal Injury Research Center (BASIR), Tehran University of Medical Sciences, Tehran, Iran and ${ }^{2}$ Nutrition Department, Iran University of Medical Sciences, Tehran, Iran

Correspondence: Dr H Sabour, Brain and Spinal Injury Research Center (BASIR), Tehran University of Medical Sciences, Imam Hospital, Keshavarz boulevard, Gharib street, Tehran 14114, Iran.

E-mail: hsabour@farabi.tums.ac.ir

Received 26 November 2013; revised 12 February 2014; accepted 18 February 2014; published online 18 March 2014 
Inclusion criteria were: traumatic SCI and post injury duration longer than 1 year. All patients who entered the investigation had traumatic SCI. We selected those in stable phase of SCI (time since injury longer than 1 year) so that the changes of patients' disability and dependency scores could be related to omega-3 PUFA administration and not the recovery process from other accident-induced complications. Patients were excluded based on exclusion criteria (pregnancy, lactation, any rehabilitation therapy, amputation and nontraumatic SCI etiology, history of diabetes, cancer, endocrinology disease, acute infection, use of special medications such as glucocorticoids, thyroid hormones, GnRH analogs, anticonvulsive drugs, heparin, aluminum containing antiacids, lithium, omega 3 fatty acids or other nutrients supplements). Data were collected from November 2010 to April 2012. The protocol was approved by the ethics committee at Tehran University of Medical Sciences (Approval number: 1421 at 18 July 2010).

\section{Study design}

Fifty patients in control group and 54 patients in treatment group were randomized by using Permuted Balanced Block Randomization Method. Treatment group received two MorDHA capsules ( $435 \mathrm{mg}$ of docosahexaenoic acid and $65 \mathrm{mg}$ of eicosapentaenoic acid) per day and patients in control group received two placebo capsules which were consumed twice daily. No specific advices on food intake were given to patients and no diet modification was made by us through the study. We used pill count method for assessment of compliance based on a confidence relationship between patient and physician. Patients were not given specific advice on food intake during the study. No lifestyle, diet or medication changes were recorded at every 4 weeks' phone calls or face-to-face interviews.

Omega 3 placebo capsules were provided by Minami Nutrition Co. (Aartselaar, Belgium) and placebo capsules were supplied by Zahravi Pharmaceutical Co. (Tabriz, Iran). Both capsules were similar in color, shape, and taste. Calcium capsules were provided by Darou Pakhsh Pharm Co. (Tehran, Iran) and consisted $1000 \mathrm{mg}$ calcium and $400 \mathrm{IU}$ vitamin D. Both groups of treatment and control received calcium capsules (one capsule daily).

Data were collected at the beginning of the study and after 14 months of $\omega$-3 PUFAs consumption.

\section{FIM + FAM scores}

Here, we used FIM and FAM (FIM + FAM $)^{7}$ to assess changes of disability and dependency scores after omega-3 PUFA administration.

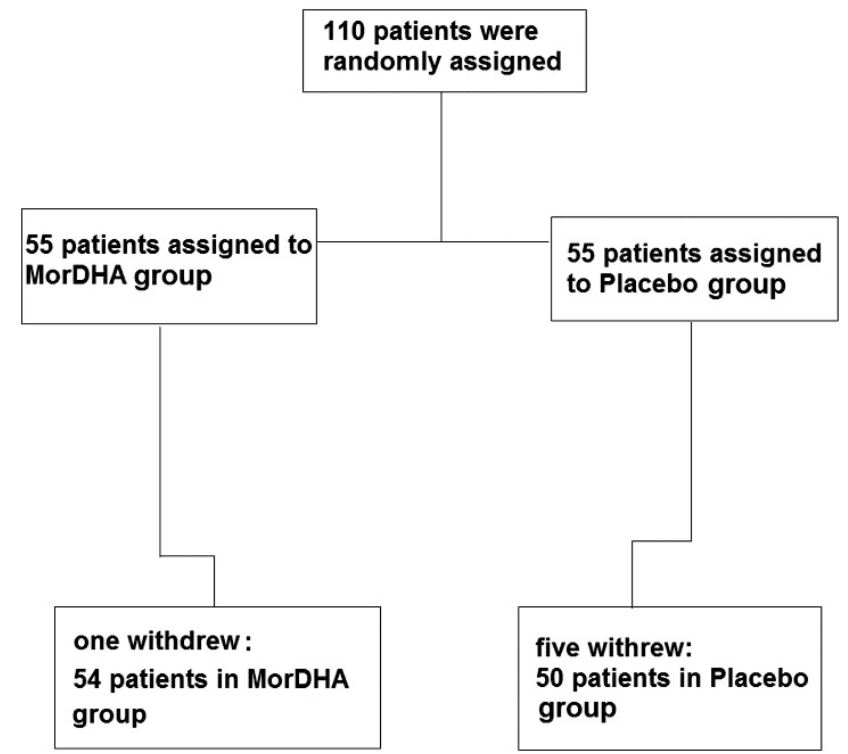

Figure 1 The drop out numbers of spinal cord-injured patients assigned to each group of treatment and placebo group through 14 months of trial.
FIM includes 18 items in which 13 items represent motor function and 5 items epitomize cognitive status. ${ }^{8}$ FAM adds 12 further items to FIM (total items of 30) to address functional areas that are less emphasized in FIM including behavioral, cognitive and communication measures. The original US version of FAM was developed in 1990s mostly to assess outcomes after brain injury. ${ }^{9}$ The UK version of FIM + FAM showed improved reliability in comparison with previous US version. ${ }^{10}$ This scale contains 18 items of FIM and 13 items of FAM which are shown in Supplementary Appendix 1. Each item is scored from 1-7 in which level 1 represents 'total dependency' and levels 2-4 illustrate maximal, moderate and minimal assistance necessity, respectively. Level 5 epitomizes 'supervision or set-up' which shows that patient only needs help for equipment set-up. Level 6 and 7 represent 'modified' and 'complete' independence, respectively. Previously, the Iranian version of FAM (FAM-Ir) has shown an acceptable reliability in evaluating functional disability. ${ }^{11}$ The 30 items of FIM + FAM can be organized into two main domains; motor (consists of three subscales of self-care, bladder/bowel management and locomotion) and cognitive (consists of two subscales of communication and psychosocial).

\section{Statistical analyses}

All statistical analyses were performed using SPSS software, Version 18 (SPSS, Chicago, IL, USA). Categorical data are reported using percentages and continuous quantitative values are reported by mean $\pm 95 \%$ confidence interval. Median of each component score in FIM + FAM was calculated. Proper comparison of means was performed using $t$-test and one-way analysis of variances (ANOVA) before and after intervention. $P<0.05$ was considered statistically significant. Multiple correction tests were applied using BenjaminiHochberg procedure to correct $P$-values for occurrence of false positives with $\mathrm{R}$ statistical software version 3.0.2.

\section{Statement of ethics}

We certify that all applicable institutional and governmental regulations concerning the ethical use of human volunteers were followed during the course of this research.

\section{RESULTS}

One-hundred and four patients entered in this study in which gender distribution showed dominant frequency of male individuals $(18.3 \%$ ( $n: 19)$ females and $81.7 \%$ ( $n: 85)$ males). Treatment group consisted of 54 patients with mean age of $54.12 \pm 11.76$ years and control group included 50 patients with mean age of $51.15 \pm 13.43$ years. Mean post

Table 1 Demographic characteristics in spinal cord-injured patients; no significant difference was detected between treatment and placebo groups

\begin{tabular}{lccc}
\hline Feature & $\begin{array}{c}\text { Treatment group } \\
\text { (n: } 54)\end{array}$ & $\begin{array}{c}\text { Placebo group } \\
\text { (n: } 50)\end{array}$ & P-value \\
& & & \\
\hline Gender distribution & & & \\
$\quad$ Male & $81.5 \%(n: 44)$ & $82 \%(n: 41)$ & 0.57 \\
Female & $18.5 \%(n: 10)$ & $18 \%(n: 9)$ & \\
& & & \\
Injury level & & & \\
$\quad$ Cervical & $25.9 \%(n: 14)$ & $14(n: 7)$ & 0.29 \\
$\quad$ Thoracic & $59.3 \%(n: 32)$ & $66(n: 33)$ & \\
Lumbar & $14.8 \%(n: 8)$ & $20(n: 10)$ & \\
Mean age \pm s.d. (year) & $51.15 \pm 13.43$ & $54.12 \pm 11.76$ & 0.23 \\
Time since injury \pm s.d. (year) & $8.96 \pm 5.44$ & $9.56 \pm 7.20$ & 0.63 \\
Mean weight \pm s.d. (kg) & $68.78 \pm 15.15$ & $68.84 \pm 13.87$ & 0.98 \\
Mean height \pm s.d. (cm) & $168.67 \pm 9.194$ & $170.32 \pm 9.08$ & 0.35 \\
Mean body mass index & $24.119 \pm 4.89$ & $23.64 \pm 3.78$ & 0.58 \\
\pm s.d. (kg m ${ }^{-2}$ ) & & &
\end{tabular}


injury duration was $8.96 \pm 5.44$ years (range: $1-31$ ) in treatment group and $9.56 \pm 7.20$ (range: 1-33) in placebo group. Patients' demographic features in treatment and placebo groups are shown in Table 1. There was no significant difference in mean age, weight, height, body mass index and injury level between treatment and control groups.

Compliance with supplement averaged $80 \%$ in both groups over the 14 months of observation. No significant effect of omega-3 PUFA consumption could be detected on any components of FIM + FAM after 14 months of omega 3 fatty-acid consumption.

The outcomes of FIM + FAM in main domains and subscales are shown in Table 2. Two groups of treatment of placebo revealed no significant difference in these scores. After 14 months of $\omega-3$ PUFA administration, no noticeable changes was detected in FIM + FAM results. Figures 2 and 3 illustrate radar chart of FIM + FAM scores in treatment and placebo group.

The lowest scores were detected in 'motor' domain. Locomotion subscale showed to impose the most severe disability. Bladder/bowl management along with self-care components played second and third roles in patients' dependency. Most patients were completely independent in cognitive domain. Communication subscale attained the highest UK FIM + FAM scores.

\section{DISCUSSION}

In this study, we observed that consumption of $\omega-3$ PUFA in patients with chronic SCI does not improve ability and has no significant advantage in rehabilitation process.

There are persuasive evidences showing that omega-3 PUFA modulates neurological function through multitude overlapping mechanisms. ${ }^{12}$ Its probable positive effects have been shown on Alzheimer's disease ${ }^{13}$ and Huntington ${ }^{14}$ in human models, however, its effect on acute neural injury such as acute traumatic SCI have been only investigated on experimental laboratory models and have shown promising outcomes. ${ }^{4,15,16}$

Previously, neuroprotective effect of omega 3 PUFAs was observed in acute phase of SCI. ${ }^{4,16,17}$ Most of these studies support this positive

Table 2 Domain and subscale scores before and after intervention with omega-3 polyunsaturated fatty acids in spinal cord-injured patients

\begin{tabular}{|c|c|c|c|c|c|c|}
\hline & \multicolumn{3}{|c|}{ Before Intervention } & \multicolumn{3}{|c|}{ After Intervention } \\
\hline & \multirow{2}{*}{$\begin{array}{c}\text { Treatment group } \\
\text { Mean } \pm \text { s.d. (Range) }\end{array}$} & \multicolumn{2}{|l|}{ Placebo group } & \multirow{2}{*}{$\begin{array}{c}\text { Treatment group } \\
\text { Mean } \pm \text { s.d. (Range) }\end{array}$} & \multicolumn{2}{|l|}{ Placebo group } \\
\hline & & Mean \pm s.d. (Range) & P-value & & Mean \pm s.d. (Range) & P-value \\
\hline \multicolumn{7}{|l|}{ Domains } \\
\hline Motor (16-112) & $77.67 \pm 20.31(27-105)$ & $83.57 \pm 21.65(31-111)$ & 0.16 & $78.93 \pm 19.42(22-105)$ & $84.13 \pm 22.74(28-112)$ & 0.25 \\
\hline Cognitive (14-98) & $91.07 \pm 6.34(72-98)$ & $92.60 \pm 6.25(76-98)$ & 0.24 & $91.13 \pm 6.50(65-97)$ & $91.95 \pm 10.22$ (39-98) & 0.65 \\
\hline \multicolumn{7}{|l|}{ Subscale } \\
\hline Self-care (7-49) & $39.88 \pm 10.13(13-49)$ & $41.77 \pm 9.82(13-49)$ & 0.34 & $39.88 \pm 10.13(13-49)$ & $41.77 \pm 9.82(13-49)$ & 0.34 \\
\hline $\begin{array}{l}\text { bladder/bowel management } \\
(2-14)\end{array}$ & $10.28 \pm 2.58(2-14)$ & $11.10 \pm 2.45(2-14)$ & 0.10 & $10.43 \pm 3.13(2-14)$ & $11.45 \pm 2.32(2-14)$ & 0.08 \\
\hline Locomotion (7-49) & $27.50 \pm 11.27(7-48)$ & $30.72 \pm 12.03(7-48)$ & 0.17 & $27.90 \pm 10.98(7-49)$ & $30.62 \pm 12.29(7-49)$ & 0.28 \\
\hline Psychosocial (9-63) & $56.17 \pm 6.25(37-63)$ & $57.56 \pm 6.18(41-63)$ & 0.27 & $56.80 \pm 5.16(43-62)$ & $57.68 \pm 6.86(34-63)$ & 0.50 \\
\hline Communication (5-35) & $34.98 \pm 0.13(34-35)$ & $35.0 \pm 0.00(35-35)$ & 0.34 & $34.34 \pm 4.42(5-35)$ & $34.31 \pm 4.52(5-35)$ & 0.97 \\
\hline Total & $168.23 \pm 25.23(106-201)$ & $175.62 \pm 26.42(108-209)$ & 0.16 & $170.13 \pm 23.37(100-200)$ & $176.34 \pm 30.96(67-210)$ & 0.29 \\
\hline
\end{tabular}

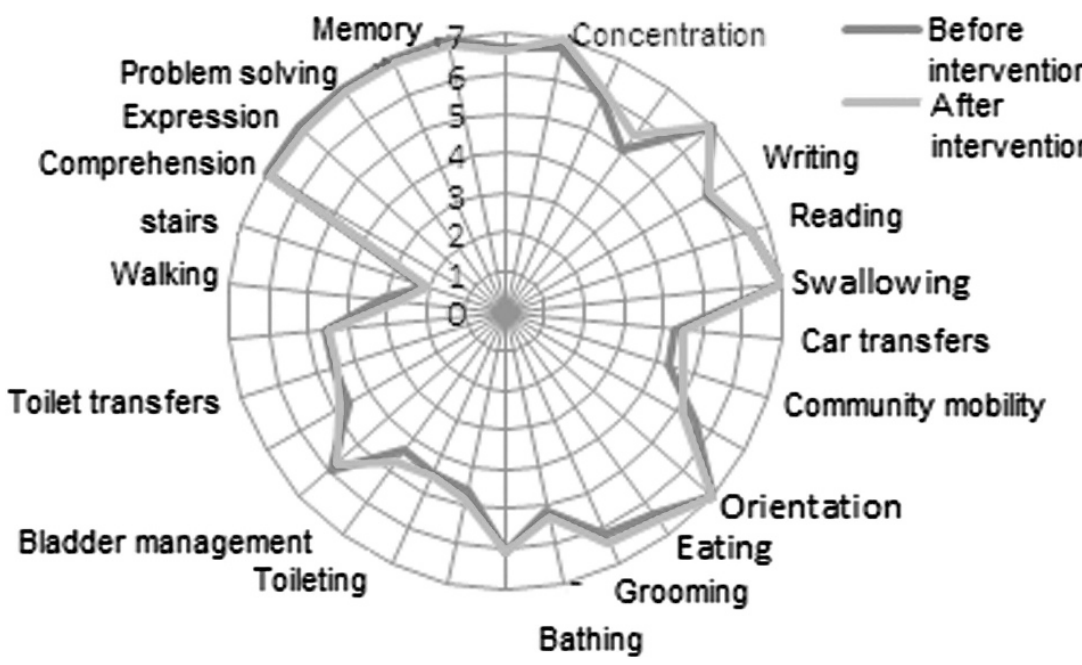

Figure 2 Radar chart of Functional Independence Measure and Functional Assessment Measure (FIM +FAM) scores in placebo group in spinal cord-injured patients. 


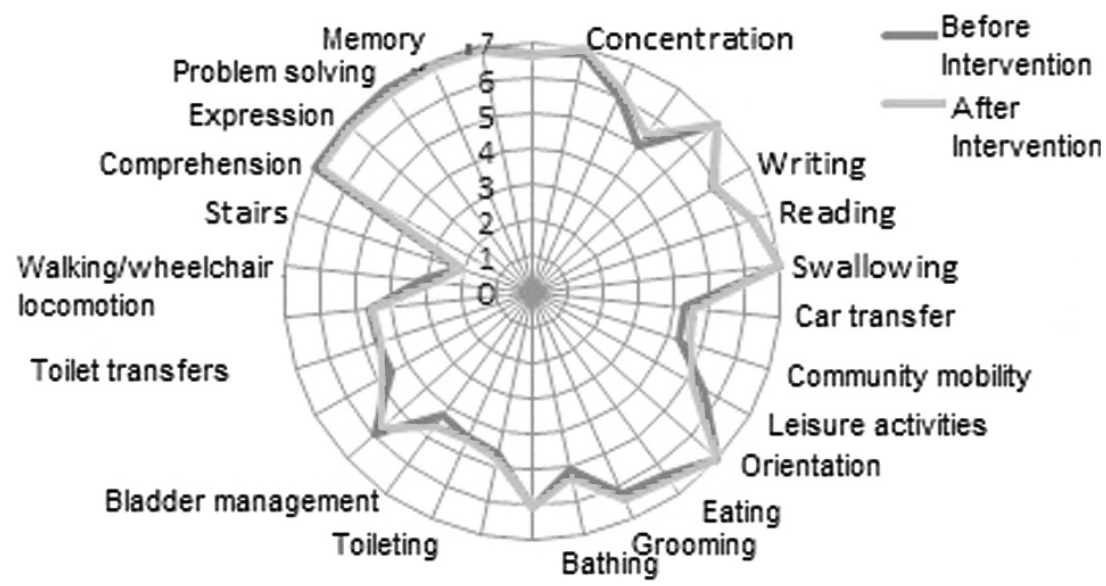

Figure 3 Radar chart of Functional Independence Measure and Functional Assessment Measure (FIM + FAM) scores in treatment group in spinal cord-injured patients.

effect on experimental animal models, while literatures on human models are so limited. By considering the proposed mechanisms of $\omega-3$ PUFA in induction of neurorecovery, which originate mostly from its anti-oxidative and anti-inflammatory effects, ${ }^{18}$ it is expectable to observe noticeable influence in acute phase of injury when these inflammatory reactions are at their peak level. Previous literatures has supported the fact that a state of chronic inflammation occurs after SCI ${ }^{19,20}$ and this steady inflammation even explained higher risk of atherogenic events in these patients. ${ }^{20}$ Some studies have also demonstrated that alterations in immune system in spinal cord-injured patients may contribute in this low-grade inflammatory process. ${ }^{21}$ Theoretically by considering this chronic inflammation state in individuals with SCI, we expect to observe some degrees of improvement by administration of anti-inflammatory agents. However, our study does not support the positive effect of $\omega-3$ PUFA in reduction of inflammation and subsequently neuroprotection in chronic phases of SCI. Although critical medical condition in patients with SCI in acute phase limits designing investigations to assess effects of $\omega-3$ PUFA, studies on mice showed significant neural improvement and partial recovery from complete paralysis ${ }^{22,23}$ in this phase of SCI.

It seems that neuroprotective effect of $\omega-3$ PUFA in patients with chronic SCI, if exists, is very slight and cannot exert a significant influence of disability and independency measures. Moreover, the effect of $\omega-3$ PUFA consumption on inflammatory cytokines after 4 months of intervention was investigated previously and the results demonstrated no significant alteration. ${ }^{24}$ Here, we propose that omega-3 PUFA administration in chronic traumatic SCI does not result in neuroprotection and its anti-inflammatory effect in this phase, if exists, cannot clinically change FIM + FAM scores.

FIM + FAM scale is a semi-verbal assessing method for evaluating disability in which many of domains are assessed verbally and confirmed by observation. Outcomes of FIM + FAM are reliable and sensitive to intervention induced changes. ${ }^{25}$ However, some components especially cognitive scales have shown to change only in small amounts ${ }^{26}$, which was described by a ceiling effect identified by van der Putten et al. ${ }^{27}$ in 1999 . In our study, most patients in treatment and control groups attained the highest scores in cognitive domain (psychosocial and communication subscales) before intervention and these scores showed no noticeable changes after trial. As it was expected, the lowest scores was observed in locomotion subscale and secondly in sphincter control in patients with SCI.
These scores also showed no significant changes after 14 months of $\omega-3$ PUFA consumption.

\section{Study limitations and further investigations}

As omega-3 PUFA has anti-inflammatory effects, it is expected to observe its efficacy mostly in acute phase of SCI when the inflammation processes are more severe. However, limited access to patients with traumatic SCI in acute phase (as they are mostly admitted in ICU which complicates oral administration of omega-3 PUFA) and co-existence of accident-induced complications in this phase, which interferes with our interpretations of patients' disability and dependency, made us to perform this investigation on patients with stable SCI in chronic phase. However, studies on acute phase of SCI may enlighten the neuroprotective effects of omega-3 fatty acids and can be compared with our results. Moreover, here we evaluated disability and dependency with FIM + FAM measurement. Our study showed no effect of omega-3 PUFA on FIM + FAM scores and the effect of its administration on other dependency measurement instruments in SCI such as spinal cord independence score (SCIM) can be used in future investigations to confirm our results.

\section{CONCLUSION}

Although omega-3 fatty acids have shown to have neuroprotective effect in acute phase of SCI, it seems it has no significant influence on disability and dependency scores in chronic inflammatory state of SCI. The positive effect of $\omega-3$ fatty acid in chronic neurorecovery process, if exists, is weaker to exert any significant improvement in UK FIM + FAM scores.

\section{DATA ARCHIVING}

There were no data to deposit.

\section{CONFLICT OF INTEREST}

The authors declare no conflict of interest.

\section{ACKNOWLEDGEMENTS}

This study was supported by Tehran University of Medical Sciences. We appreciate the help of all staffs in recruiting process and data collection. We also like to thank Dr Mohammad Reza Vafa for his valuable consultations in the process of designing the study. This study is part of $\mathrm{Ph}$. D project supported by a grant from Tehran University of Medical Sciences (Tehran, Iran). 
1 Middleton J, Tran Y, Craig A. Relationship between quality of life and self-efficacy in persons with spinal cord injuries. Arch Phys Med Rehabil 2007; 88: 1643-1648.

2 Go BK, DeVivo MJ, Richards JS. The Epidemiology Of Spinal Cord Injury. In: Stover SL, DeLisa JA and Whiteneck GG (eds). Spinal cord injury. Clinical outcomes from the model systems. Aspen Publishers: Gaithersburg, 1995, pp 21-55.

3 Rahimi-Movaghar V, Saadat S, Rasouli MR, Ganji S, Ghahramani M, Zarei MR et al. Prevalence of spinal cord injury in Tehran, Iran. J Spinal Cord Med 2009; 32: 428-431.

4 King VR, Huang WL, Dyall SC, Curran OE, Priestley JV, Michael-Titus AT. Omega-3 fatty acids improve recovery, whereas omega- 6 fatty acids worsen outcome, after spinal cord injury in the adult rat. J Neurosci 2006; 26: 4672-4680.

5 Lang-Lazdunski L, Blondeau N, Jarretou G, Lazdunski M, Heurteaux C. Linolenic acid prevents neuronal cell death and paraplegia after transient spinal cord ischemia in rats. J Vasc Surg 2003; 38: 564-575.

6 Endres S, von Schacky C. n-3 polyunsaturated fatty acids and human cytokine synthesis. Curr Opin Lipidol 1996; 7: 48-52.

7 Hall KM, Hamilton BB, Gordon WA, Zasler ND. Characteristics and comparisons of functional assessment indices: Disability Rating Scale, Functional Independence Measure, and Functional Assessment Measure. J Head Traum Rehabil 1993; 8: 60-74.

8 Linn RT, Blair RS, Granger CV, Harper DW, O'Hara PA, Maciura E. Does the Functional Assessment Measure (FAM) extend the functional independence measure (FIM) instrument? A Rasch analysis of stroke inpatients. J Outcome Meas 1999; 3: 339-359.

9 Hobart JC, Lamping DL, Freeman JA, Langdon DW, McLellan DL, Greenwood RJ et al. Evidence-based measurement: which disability scale for neurologic rehabilitation? Neurology 2001; 57: 639-644.

10 Donaghy S, Wass PJ. Interrater reliability of the Functional Assessment Measure in a brain injury rehabilitation program. Arch Phys Med Rehabil 1998; 79: 1231-1236.

11 Hadian MR, Yekaninejad MS, Salehin F, Razavi SH, Javidan AN, Pakpour AH et al. Cross-cultural adaptation and reliability evaluation of Iranian version of Functional Assessment Measure in spinal cord injury patients. Neurol Neurochir Pol 2012; 46: 351-356.

12 Dyall SC, Michael-Titus AT. Neurological benefits of omega-3 fatty acids. Neuromolecular Med 2008; 10: 219-235.

13 Freund-Levi Y, Eriksdotter-Jönhagen M, Cederholm T, Basun H, Faxén-Irving G, Garlind A et al. Omega-3 fatty acid treatment in 174 patients with mild to moderate Alzheimer disease: OmegAD study: a randomized double-blind trial. Arch Neurol 2006; 63: 1402-1408.

14 Puri BK, Bydder GM, Counsell SJ, Corridan BJ, Richardson AJ, Hajnal JV et al. MRI and neuropsychological improvement in Huntington disease following ethyl-EPA treatment. Neuroreport 2002; 13: 123-126.
15 Michael-Titus AT, Priestley JV. Omega-3 fatty acids and traumatic neurological injury: from neuroprotection to neuroplasticity? Trends Neurosci 2014; 37: 30-38.

16 Huang WL, King VR, Curran OE, Dyall SC, Ward RE, Lal N et al. A combination of intravenous and dietary docosahexaenoic acid significantly improves outcome after spinal cord injury. Brain 2007; 130 (Pt 11), 3004-3019.

17 Lim SN, Huang W, Hall JC, Ward RE, Priestley JV, Michael-Titus AT. The acute administration of eicosapentaenoic acid is neuroprotective after spinal cord compression injury in rats. Prostaglandins Leukot Essent Fatty Acids 2010; 83: 193-201.

18 Mori TA, Beilin LJ. Omega-3 fatty acids and inflammation. Curr Atheroscler Rep 2004 6: 461-467.

19 da Silva Alves E, de Aquino Lemos V, Ruiz da Silva F, Lira FS, Dos Santos RV, Rosa JP et al. Low-grade inflammation and spinal cord injury: exercise as therapy? Mediators Inflamm 2013; 2013: 971841.

20 Wang TD, Wang YH, Huang TS, Su TC, Pan SL, Chen SY. Circulating levels of markers of inflammation and endothelial activation are increased in men with chronic spinal cord injury. J Formos Med Assoc 2007; 106: 919-928.

21 Campagnolo DI, Keller SE, DeLisa JA, Glick TJ, Sipski ML, Schleifer SJ. Alteration of immune system function in tetraplegics. A pilot study. Am J Phys Med Rehabil 1994; 73: 387-393.

22 Lim SN, Huang W, Hall JC, Michael-Titus AT, Priestley JV. Improved outcome after spinal cord compression injury in mice treated with docosahexaenoic acid. Exp Neurol 2013; 239: 13-27.

23 Satkunendrarajah K, Fehlings MG. Do omega-3 polyunsaturated fatty acids ameliorate spinal cord injury?: Commentary on: Lim et al, Improved outcome after spinal cord compression injury in mice treated with docosahexaeonic acid. Exp. Neurol. Jan 239:13-27. Exp Neurol 2013; 249: 104-110.

24 Sabour H, Larijani B, Vafa MR, Hadian MR, Heshmat R, Meybodi HA et al. The effects of $n-3$ fatty acids on inflammatory cytokines in osteoporotic spinal cord injured patients: A randomized clinical trial. J Res Med Sci 2012; 17: 322-327.

25 Dutta D, Vanere P, Gupta T, Munshi A, Jalali R. Factors influencing activities of daily living using FIM-FAM scoring system before starting adjuvant treatment in patients with brain tumors: results from a prospective study. J Neurooncol 2009; 94 . 103-110.

26 Fioravanti AM, Bordignon CM, Pettit SM, Woodhouse LJ, Ansley BJ. Comparing the responsiveness of the assessment of motor and process skills and the functional independence measure. Can J Occup Ther 2012; 79: 167-174.

27 van der Putten JJ, Hobart JC, Freeman JA, Thompson AJ. Measuring change in disability after inpatient rehabilitation: comparison of the responsiveness of the Barthel index and the Functional Independence Measure. J Neurol Neurosurg Psychiatry 1999; 66: $480-484$.

Supplementary Information accompanies this paper on the Spinal Cord website (http://www.nature.com/sc) 